\title{
Danno tanatologico: il dibattito sulla risarcibilità del danno da perdita della vita
}

\author{
Mauro Intagliata ${ }^{\bullet}$
}

\section{Riassunto}

L'Autore esamina e critica la sentenza delle SezioniUnite. $n^{\circ} 15350$ del 22/7/2015 che, chiamata ad affrontare il quesito circa la risarcibilità o meno del danno da perdita della vita immediatamente conseguente alle lesioni derivanti da fatto illecito, ha optato in favore della tesi maggioritaria e prevalente di segno negativo spegnendo le speranze sorte dalla coraggiosa sentenza "Scarano" n’1361/2014.

Ritiene infatti che il mancato riconoscimento della risarcibilità del danno "tanatologico", così come argomentato e motivato dalle S.U., non consente affatto di ritenere chiusa la questione dogmatica ed interpretativa controversa. Con appena dieci pagine di motivazione, le S.U. intenderebbero porre fine alla vexata quaestio lasciando tuttavia privo di tutela il diritto alla vita, il diritto principe della esistenza umana.

\section{Résumé}

L'auteur examine et critique le jugement $n^{\circ} 15350$ du 22/7/2015 rendu par la Cour de Cassation italienne, Sections Unies. La Cour a dû s'exprimer sur la question liée à l'indemnisation du préjudice pour son propre décès à la suite des lésions corporelles provoquées par un crime. Elle a opté pour la thèse dominante et négative qui a éteint les espoirs nés après le courageux jugement $n^{0} 1361 / 2014$ «Scarano» .

L'auteur estime en effet que la non-reconnaissance de l'indemnisation du dommage "thanatologique », ainsi que l'expliquent les Sections Unies, ne permet pas de considérer comme terminé le débat controversé dogmatique et interprétatif. Avec dix pages de motifs seulement, les Sections Unies auraient l'intention de mettre fin à cette question ainsi ancienne que controversée, sans toutefois protéger le droit à la vie, le droit fondamental de l'existence humaine.

\section{Abstract}

The author examines and criticizes the court judgment of Italian Court of Cassation, United Sections, n. 15350 of 22 July 2015 when having to deal with the issue about the compensation in the event of wrongful death as an immediate consequence of the injuries due to an illegal action, the option was for the prevailing thesis which was negative. In this way the hopes produced by the brave Scarano judgment n. 1361/2014 were cancelled.

The author underlines that the non-recognition of compensation for thanatological damage as explained by United Sections does not allow to consider the dogmatic contentious issue as concluded. With just ten written pages, they intend to explain and to finish such a vexata quaestio thus leaving the right to life without protection, that is to say the basic right of human existence.

Key words: thanatological damage; compensation; wrongful death; Scarano judgment n. 1361/2014; judgment of Italian Court of Cassation n. 15350/2015.

\footnotetext{
- E' iscritto all'Albo degli Avvocati presso l'Ordine di Reggio Emilia dal 1998. Dopo una qualificata esperienza professionale nel settore del Diritto assicurativo, ha fondato con Marta Rovacchi e Domenico Intagliata lo Studio Rovacchi Intagliata. Ha assistito, tra l'altro, alcune famiglie delle vittime del disastro ferroviario di Crevalcore del gennaio 2005.
} 
Per chi si occupa dello studio a livello giuridico delle vittime di fatti illeciti i recenti pronunciamenti della Corte di Cassazione sul danno tanatologico hanno il pregio di porre al centro dell'attenzione del dibattito dottrinario e giurisprudenziale, involgendo anche aspetti di natura filosofica ed etica, il tema del diritto al risarcimento del danno per la perdita della vita ad opera di terzi.

La questione è di grande attualità e complessità e merita quindi di essere approfondita partendo proprio dagli ultimi sviluppi giurisprudenziali.

Le Sezioni Unite della Cassazione Civile, investite del compito di risolvere il contrasto sorto tra la “rivoluzionaria" sentenza della III Sezione Civile della Cassazione nº 1361 del 23/1/2014 e l'orientamento tradizionale maggioritario in senso contrario della giurisprudenza pregressa, in virtù dell'ordinanza $n^{\circ} 5056$ del 04/3/2014 emessa dalla terza sezione civile, hanno sancito il principio di diritto dell'irrisarcibilità del danno c.d. "tanatologico" o danno da perdita della vita, trasmissibile iure successionis, producendo, tuttavia, l'effetto di riaprire e non certo di chiudere il dibattito sull'argomento.

L'impressione che si ricava dalla lettura della sentenza delle Sezioni Unite $n^{\circ} 15350$ è che il mancato riconoscimento della risarcibilità del danno "tanatologico", così come argomentato e motivato dalle S.U., non consente di giungere ad una conclusione definitiva. Con appena dieci pagine di motivazione (a fronte delle oltre cento della sentenza n ${ }^{\circ} 1361$ del 23/1/2014, detta sentenza "Scarano" dal nome del consigliere relatore) le S.U. intenderebbero porre fine alla vexata quaestio lasciando tuttavia privo di tutela il diritto alla vita, il diritto principe della esistenza umana.

Per meglio comprendere il senso del dibattito e della importanza della questione occorre preliminarmente chiarire cosa si intenda con la locuzione danno "tanatologico" e quale sia la cornice entro cui tale voce di danno ha trovato un suo spazio all'interno della generale e complessa materia del risarcimento del danno alla persona, rispetto ad altre figure contigue rappresentate dal danno biologico terminale e dal danno catastrofale

\section{Nozione di danno "tanatologico".}

Per "danno tanatologico" si intende il danno da violazione del diritto alla vita fatto valere iure successionis dagli eredi della vittima primaria dell'illecito deceduta immediatamente a seguito di lesioni mortali o che sia rimasta in vita per un breve lasso di tempo dall'evento lesivo in condizioni di incoscienza tale da escludere che la stessa possa aver percepito la fine imminente (assenza del c.d. spatium vivendi) (cfr. per tutte Cass. Civ., S.U., 11/11/2008 n. 26972); danno ritenuto non risarcibile da una granitica giurisprudenza di legittimità consolidatasi a partire dalla sentenza quasi centenaria del 1925 della Cassazione a S.U. n 3475. Tale definizione, elaborata dalla giurisprudenza in assenza di alcuna previsione normativa, viene invero più compiutamente approfondita dalla sentenza "Scarano" n. 1361/2014, ove si afferma che il danno non patrimoniale da perdita della vita consiste nella perdita del bene vita, bene supremo dell'individuo oggetto di un diritto assoluto e inviolabile garantito dall'ordinamento in via primaria, anche sul piano della tutela civile e che, sebbene appaia inconfutabile che il diritto alla vita sia altro e diverso dal diritto alla salute quest'ultima rappresentando un minus rispetto alla prima, che ne costituisce il presupposto- ciò non comporta necessariamente la conclusione che della perdita della vita debba negarsi la ristorabilità. 
Si tratta di danno altro e diverso, in ragione del diverso bene tutelato, dal danno alla salute e si differenzia pertanto dal danno biologico terminale e dal danno morale terminale (o catastrofale o catastrofico) della vittima, rilevando ex se, nella sua oggettività di perdita del bene vita, oggetto di un diritto assoluto e inviolabile.

\section{Le due categorie di danno distinte dal danno tanatologico : il danno biologico terminale o da morte e il danno catastrofale.}

Le due fattispecie di danno biologico terminale e di danno catastrofale, anch'esse di costruzione meramente giurisprudenziale, rientrano nell'alveo dei danni indicati genericamente come conseguenza del decesso della vittima primaria e attraverso una loro corretta individuazione si perviene al risultato di delimitare compiutamente il campo di applicabilità della categoria del danno tanatologico senza incorrere in errate sovrapposizioni o elisioni.

Detta distinzione rileva in particolare dal momento che è solo per queste due categorie di danno che viene riconosciuta la risarcibilità iure bereditatis.

Per quanto riguarda il danno biologico terminale esso è considerato pacificamente risarcibile iure successionis e si ha quando, a seguito delle lesioni dell'integrità fisica con esito letale, il decesso sia sopraggiunto dopo un apprezzabile lasso di tempo, potendosi concretamente configurare un'effettiva compromissione dell'integrità psicofisica del soggetto leso medicalmente accertabile e quindi liquidabile secondo i criteri applicabili alla quantificazione del danno biologico (vedi in particolare: Cass. Civ. del 21/3/2013 $\mathrm{n}^{\circ} 7126$; Cass. Civ. del 14/12/2010 n²5264; Cass. Civ. del 30/6/1998 n6404; nella giurisprudenza di merito vanno evidenziate le sentenze Trib. Bari del 16/2/2014; Trib. Monza del 12/12/2013; Trib. Roma del 3/9/2013 n¹7772).

Vi è danno biologico terminale quando si è in presenza di un danno alla salute subito dalla vittima nell'intervallo di tempo intercorrente tra la lesione e la morte, purché esso si sia protratto per una durata apprezzabile. La discrezionalità e difficoltà di connotare in modo certo ed oggettivo il requisito della "durata apprezzabile" ha spinto alcuni autori ad assegnare con toni umoristici l'appellativo di "cronometrico" al criterio di giudizio elaborato dalla giurisprudenza ${ }^{1}$.

Venendo invece al danno catastrofale o danno da lucida agonia, ritenuto anch'esso trasmissibile agli eredi, occorre rilevare che esso è costituito dalla sofferenza patita dalla vittima che, rimasta lucida all'esito della lesione, abbia vissuto l'angosciosa e consapevole attesa della propria fine imminente ed ineluttabile (vedi in particolare Cass. Civ., Sez. III, del 21/3/2013 n 7126; Cass. Civ., Sez. Lav., del 18/1/2011 nº1072 ; Cass. del 24/3/2011 nº754).

Il danno catastrofale è stato ricondotto dalle S. U. dell'11 novembre 2008 n 26972 nell'alveo del danno non patrimoniale configurando una compromissione di rilievo esclusivamente morale. I giudici del massimo consesso giurisprudenziale hanno infatti precisato che "Il giudice potrà invece correttamente riconoscere e liquidare il solo danno morale, a ristoro della sofferenza psichica provata dalla vittima di

\footnotetext{
${ }^{1}$ Vedi Cass., 19 ottobre 2007, n. 21976, in Danno resp., 313, 2008, con nota di Foffa, "Il danno non patrimoniale del soggetto in stato comatoso".
} 
lesioni fisiche, alle quali sia seguita dopo breve tempo la morte, che sia rimasta lucida durante l'agonia in consapevole attesa della fine". A differenza della figura del danno biologico terminale in cui rileva la durata apprezzabile della sopravvivenza, nel caso invece del danno catastrofale assume preminente ed esclusivo valore, non il tempo effettivo della agonia, ma la consapevolezza e l'intensità della sofferenza patita dalla vittima nell'arco, anche breve, di tempo che va dall'evento lesivo alla morte (Cass. Civ. del 24/3/2015 n5866; Cass. Civ. del 21/2013 nº7126).

Ammessa la risarcibilità dei danni come sopra definiti si può infine verificare il caso in cui le due categorie del danno biologico terminale e di quello catastrofale possano anche concorrere dando luogo ad una liquidazione del danno personalizzata e rapportata alla concreta lesione dell'integrità psico-fisica della vittima unitamente alla sofferenza soggettiva subite dalla medesima prima del decesso (cfr. ass. Civ. del 2014 n $^{\circ} 3183$; Tribunale di Grosseto del 25/5/2015 n517).

\section{Il danno tanatologico prima della "rivoluzionaria" sentenza Scarano del gennaio 2014.}

Dottrina e giurisprudenza hanno a lungo dibattuto sulla risarcibilità del danno tanatologico prima della sentenza "Scarano" del gennaio 2014 polarizzando le posizioni su due opposti fronti.

L'orientamento maggioritario e prevalente ha sempre sostenuto l'irrisarcibilità del danno da perdita della vita fondando i propri assunti sul presupposto che "il soggetto che perde la vita non è in grado di acquistare un diritto risarcitorio, perché finché è in vita non vi è perdita e quando è morto da una parte non è titolare di alcun diritto e dall'altra non è in grado di acquistarme" (Cass. Civ, Sez. III, del 23/2/2014 n 3549).

La giurisprudenza di legittimità ha mantenuto fermo tale convincimento espresso sin dal lontanissimo 1925 con la sentenza della Cassazione a S.U. $n^{\circ} 3475$ nella quale venne statuito il principio del mancato risarcimento del danno alla vita in caso di morte immediata.

Non è un caso che la recente sentenza delle S.U. n¹5350 del luglio 2015 abbia più volte fatto ricorso proprio al precedente originario pronunciamento del 1925 rimarcando in tal modo la valenza e la forza di una interpretazione giuridica antica e solida, non suscettibile di essere modificata dall'evoluzione del pensiero giuridico e sociale espresso dall'attualità del nostro tempo.

La tesi maggioritaria, supportata da una salda giurisprudenza, basa il proprio convincimento riportandosi all'insegnamento che assegna all'articolo 2043 c.c. una funzione di mera riparazione del danno.

Detta norma mira a tenere indenne il soggetto danneggiato dalle conseguenze pregiudizievoli derivanti dall'illecito attraverso la restituzione o il ristoro del patrimonio del creditore danneggiato. Proprio in considerazione di ciò il risarcimento del danno è definito come l'obbligazione volta a ristabilire nel patrimonio del danneggiato la situazione che si sarebbe registrata in assenza dell'illecito.

Alla luce di tali considerazioni l'orientamento maggioritario in giurisprudenza ha sempre escluso la possibilità di risarcire il danno tanatologico nella sua veste di danno biologico inteso nella sua più profonda gravità, quale totale e massima lesione del bene giuridico salute.

La vita e la salute -sotto il profilo giuridico- rappresentano due beni giuridici distinti e, nel caso in cui l’illecito abbia colpito il bene vita, la perdita di quest'ultima, per il definitivo venir meno del soggetto 
danneggiato, non può tradursi nel contestuale acquisto al patrimonio della vittima di un corrispondente diritto al risarcimento, trasmissibile come tale agli eredi.

Nel caso di perdita della vita si tratta, infatti, della lesione di un bene intrinsecamente connesso alla persona del suo titolare e da questi fruibile solo in natura.

Si deve pertanto escludere che il risarcimento del danno, per la sua funzione riparatoria, possa operare quando la persona abbia cessato di esistere (cfr. Cass. Civ. n 3549/2004; Cass. Civ. n²134/2000; Cass. Civ. n 491/1999; nonché Corte Costituzionale n 372/1994).

In caso contrario si giungerebbe alla conclusione di assegnare alla tutela dell'articolo 2043 c.c. una funzione solo sanzionatoria, non ammissibile ed incompatibile con l'intero sistema del risarcimento del danno elaborato dalla giurisprudenza.

L'orientamento contrario, condiviso in alcune sentenze di merito e soprattutto in dottrina, sebbene minoritario, prendendo le distanze dalle argomentazioni sopra riportate, fonda le proprie tesi ponendo al centro della questione l'identità ontologica della vita e della salute. Non si tratta di due entità diverse ma di due facce della stessa moneta, ovvero l'integrità ed incolumità della persona.

Tale orientamento contesta la dicotomia tra diritto alla vita e diritto alla salute sostenuto dai fautori della tesi dominante, secondo cui i due diritti menzionati avrebbero una strutturazione ontologica autonoma ed indipendente, quali categorie soggettive distinte.

Invero, la vita e la salute, per i sostenitori della tesi minoritaria, sia pur potendo essere valutate sotto profili diversi, sono dimensioni dell'essere umano assolutamente connesse ed imprescindibili l'una dall'altra.

La salute è solo un modo per definire la vita ed il diritto alla salute, in una visione costituzionale, altro non è che l'estrinsecazione della vita che si manifesta nella sua potenzialità biologica.

In definitiva, la compromissione della vita non può considerarsi fatto ontologicamente diverso rispetto alla compromissione della salute. Una compromissione della salute può comunque aversi in maniera parziale, senza arrivare alla conseguenza dell'evento morte, ma quando la lesione alla salute, alla integrità biologica è totale, allora la conseguenza lesiva sarà quella della compromissione totale, definita come morte biologica Alcune sentenze di merito nel corso degli ultimi decenni, andando contro l'orientamento granitico contrario della giurisprudenza di legittimità, riportandosi ai principi menzionati si sono espresse a favore del risarcibilità del danno da perdita della vita:

-Tribunale di Massa Carrara sentenza 20/1/1990: "Nel momento in cui l'integrità psicofisica di una persona (situazione giuridica iniziale) viene negativamente mutata dall'azione od omissione dolosa o colposa di un altro soggetto, entra nel patrimonio del leso il diritto al risarcimento del danno e, se la lesione giunge ad una gravità tale da determinare la morte del leso stesso (situazione finale), detto credito risarcitorio, che forma e concorre a formare l'asse, si trasmette iure ereditario agli aventi diritto. Tale azione ha una sua progressività anche quando sembra che la morte sia istantanea. L'evento, che è conseguenza dell'azione od omissione e che chiude il fatto antigiuridico, va dalle semplici contusioni, alla menomazione, sino alla vitale disaggregazione dell'organismo con conseguente morte del soggetto".

- Tribunale di Napoli sentenza 6/2/1991 n³86: "la contraria opinione (relativa alla non configurabilità del danno da morte immediata), la quale, pur riconoscendo il diritto al risarcimento in caso di compromissione del bene salute in dipendenza di lesioni non letali, pretende, poi, di precludere il risarcimento medesimo alla vittima di lesioni che provochino la 
morte, appare inficiato da inammissibile incongruenza logica e postula un'interpretazione in evidente contrasto con i principi costiturionali che tutelano i fondamentali beni e valori personali, come posti in rilievo anche dalla stessa Corte cost. (sent. $n$. 184/1986)".

-Tribunale di Macerata sentenza 26/7/20022: "il danneggiato, nel momento in cui diviene vittima di una lesione mortale ed ove tale lesione sia derivata da una condotta ingiusta ex art. 2043 c.c., ha già acquisito, nel proprio patrimonio, il diritto a vedere risarcita l'ingiusta menomaz̧ione della propria integrità psico-fisica e poco importa che la morte sopraggiunga istantaneamente o ad apprezzabile distanza di tempo, perché il credito risarcitorio è già sorto e, in quanto tale, è una entità giuridicamente ed economicamente apprezabile, trasmissibile agli eredi, come tutti gli assetti patrimoniali del de cuius".

Da tali pronunciamenti si può concludere che l'esigenza di tutelare il diritto alla vita, come sopra evidenziato, rappresenta il presupposto implicito di ogni situazione giuridica soggettiva riconosciuta dall'ordinamento.

A questo proposito occorre qualificare il diritto alla vita come un vero e proprio diritto soggettivo (al pari del diritto di proprietà) facente parte del patrimonio del danneggiato e la cui lesione legittima il risarcimento e la sua trasmissione agli eredi a prescindere dalla temporanea permanenza in vita della vittima.

La giurisprudenza di merito minoritaria non ritiene affatto condivisibile l'opposta tesi fondata sul rilievo che "il titolare del diritto leso non è più in vita e, quindi, non può percepire la perdita subita e non può essere titolare del credito risarcitorio che sorge dalla produzione del danno".

Secondo il Tribunale di Napoli nella citata sentenza "Stabilire chi sia titolare del credito risarcitorio è questione non già naturalistica, che si possa risolvere negativamente sulla base del fatto che il danneggiato ha perduto la vita, ma giuridica, da risolversi, dunque, secondo criteri analoghi a quelli che, sul versante opposto dell'obbligazione risarcitoria, conducono ad imputare la responsabilità risarcitoria di determinati danni a soggetti diversi da quelli che li hanno materialmente prodotti".

Il convincimento della prevalente dottrina, favorevole all'autonoma risarcibilità del danno tanatologico, è fondato sui seguenti principi:

-il diritto alla vita, in quanto fondamentale ed imprescindibile diritto dell'uomo, esige adeguata tutela; se il sistema del risarcimento del danno alla persona attribuisce rilevanza a lievi lesioni del diritto alla salute e nega tutela alla lesione del diritto alla vita si giunge inevitabilmente ad irragionevoli iniquità.

-la tutela risarcitoria è la tutela minima riconosciuta a qualunque diritto; essa, pertanto, va a maggior ragione riconosciuta al supremo ed inviolabile diritto alla vita.

-non affermare la risarcibilità del danno tanatologico porta ad un inaccettabile paradosso dal momento che, dal punto di vista del danneggiante, è più conveniente uccidere che ferire.

\section{La sentenza della Cassazione Civile del 23/1/2014 $\mathrm{n}^{\circ} 1361$ sul danno da perdita della vita, detta sentenza "Scarano".}

\footnotetext{
${ }^{2}$ In Corti marchigiane, 181, febbraio 2004.
} 
La sentenza "Scarano", in assoluta controtendenza rispetto al passato, ha invece ammesso la risarcibilità iure hereditario del danno da perdita della vita immediatamente conseguente alle lesioni derivanti da un fatto illecito.

Il principio posto a base di tale convincimento è che il danno da perdita della vita è altro e diverso, in ragione del diverso bene tutelato, dal danno alla salute, e rileva ex se nella sua oggettività di perdita del principale bene dell'essere umano a prescindere dalla consapevolezza che il danneggiato ne abbia, e deve essere ristorato anche in caso di morte cd. immediata o istantanea, senza che assumano pertanto al riguardo rilievo la persistenza in vita all'esito del danno evento da cui la morte derivi né l'intensità della sofferenza interiore patita dalla vittima in ragione della lucida percezione dell'ineluttabile sopraggiungere della propria fine.

Il diritto al ristoro del danno da perdita della vita si acquisisce dalla vittima istantaneamente al momento della lesione mortale, e quindi anteriormente all'exitus, costituendo ontologica, imprescindibile eccezione al principio dell'irrisarcibilità del danno-evento e della risarcibilità dei soli danni-conseguenza.

La morte infatti determina la perdita di tutto e non solo di qualcosa o di qualche bene che faccia parte della vita; con la morte viene meno il bene supremo della esistenza, non solo di qualche effetto o conseguenza, ma del tutto, di tutto ciò che faceva parte della vita della vittima e che avrebbe continuato a manifestarsi in tutti gli ambiti della esistenza della persona qualora il fatto illecito non ne avesse provocato la cessazione.

\section{La perdita della vita intesa come danno da perdita di chances di sopravvivenza.}

A questo proposito va rilevato che parte della dottrina ha sollevato forti perplessità quanto alla percorribilità della tesi del danno da perdita della vita, quale danno evento nella sua configurazione di eccezione alla regola della risarcibilità dei soli danni conseguenza.

Secondo tale orientamento la suddetta pronuncia non persuade e non convince laddove tenta di limitare l'eccezione al principio di irrisarcibilità del danno-evento al caso di lesione del diritto alla vita.

Le argomentazioni della sentenza Scarano su tale aspetto porrebbero le basi per un inevitabile ritorno, di carattere più complessivo, alla teoria del danno-evento, tale da comportare un sostanziale rovesciamento del sistema attualmente applicato.

Al fine di evitare tale ribaltamento occorrerebbe sottolineare che, in caso di lesione di un diritto che verte su un bene immateriale, non è l'alterazione del bene a costituire danno, ma esclusivamente le conseguenze dalla stessa scaturenti. Andrebbe posta l'attenzione, quindi, su quelle che sono le compromissioni innescate dalla lesione del bene vita in capo alla vittima. Un primo rilievo è che il danno non è rappresentato dalla morte: essa, casomai, consiste nell'evento che determina la lesione del diritto alla vita.

E come già affermato in passato in dottrina, si tratta di valutare - sul piano logico-giuridico, e non già temporale - quali siano gli effetti di tale lesione.

A questo proposito, un'indicazione importante può essere tratta da quanto si ricava in materia di perdita di chance di sopravvivenza. 
Secondo tale prospettazione ciò che rileva è la menomazione di una capacità dell'individuo, collegata al godimento del diritto alla vita. A subire una compromissione è, cioè, l'attitudine alla sopravvivenza. Va in definitiva valorizzata una capacità dell'individuo, la cui alterazione rappresenta un pregiudizio per la vittima. Più precisamente il pregiudizio che consegue all'evento-morte è rappresentato dalla distruzione della capacità di sopravvivenza dell'individuo deceduto. Non si verte in tema di danno futuro ma della compromissione di un potenziale di vita di cui un individuo gode in un determinato concreto momento storico con tutte le sue peculiarità in termini di età, sesso e condizioni di salute.

Più in generale tale tesi consente di pervenire alla conclusione che potrà essere garantita una tutela risarcitoria nei casi in cui l'attitudine o la capacità del soggetto sia stata definitivamente compromessa dall'illecito provocando la morte della vittima.

Una configurazione del genere permette di collocare anche il danno da perdita della vita nel quadro dell'attuale sistema risarcitorio, senza introdurre pericolose eccezioni.

Secondo tale ricostruzione, si può completare in maniera armoniosa il quadro della tutela del danno alla persona. In effetti, nella situazione attuale la perdita della vita trova accoglimento, ma solo entro lo schema del danno terminale, per cui non viene riconosciuta a tutte le vittime, ma soltanto a quella parte di esse per le quali si ravvisa un intervallo di sopravvivenza (danno biologico terminale) ovvero l'esistenza di uno stato di lucida consapevolezza durante l'agonia (danno catastrofale).

Come già a suo tempo osservato dalla giurisprudenza di legittimità (Cass. Civ. del 2007 n²1976), ammettere il risarcimento del danno da perdita della vita impone di rivedere l'intera materia del danno da morte, non potendo lo stesso essere sommato con i danni terminali conseguenti a periodi anche brevi di sopravvivenza: danni la cui liquidazione è palesemente attribuita in considerazione della ritenuta impraticabilità dell'altra e più radicale soluzione.

A tali pregiudizi, attualmente liquidati ad una parte delle vittime, andrebbe a sostituirsi il danno da perdita della vita, garantito a tutte le vittime di un illecito mortale, assicurando così una definitiva razionalizzazione del sistema risarcitorio.

\section{La giurisprudenza di merito post-sentenza "Scarano"sulla risarcibilità del danno da perdita della vita e la quantificazione del danno da perdita della vita.}

Va segnalato per completezza di indagine che subito dopo la rivoluzionaria sentenza Scarano alcuni giudici di merito hanno recepito il nuovo orientamento della Cassazione riconoscendo la sussistenza ed autonomia del danno da perdita della vita.

A questo proposito si segnalano: la sentenza della Corte di Appello di Cagliari $n^{\circ} 438$ del 1/7/2014; la sentenza della Corte di Appello di Milano ${ }^{\circ} 4307$ del 1/12/2014; la sentenza del Tribunale del Vallo della Lucania $n^{\circ} 158$ del 30/4/2014; la sentenza del Tribunale di Brindisi $n^{\circ} 2039$ del 1/12/2014; la sentenza del Tribunale di Piacenza n ${ }^{\circ} 97$ del 5/2/2015.

Tutte ammettono, sebbene con diverse sfumature, la risarcibilità del danno da perdita della vita riportandosi sostanzialmente ai principi stabiliti dalla sentenza "Scarano". 
Venendo poi al tema della quantificazione di tale categoria di danno occorre rilevare che l'autonomia del bene vita rispetto al bene salute/integrità psico-fisica comporta un sistema di quantificazione particolare e specifico, diverso da quello dettato per il danno biologico la cui valutazione equitativa spetta al giudice di merito ed è rimessa alla sua prudente discrezionalità l'individuazione dei criteri di relativa valutazione.

Deve ritenersi allora ammissibile qualsiasi modalità che consenta di addivenire ad una valutazione equa parametrata, non a semplici valutazioni soggettive o alla determinazione di un ammontare uguale per tutti, ma tenendo conto ad esempio dell'età delle condizioni di salute e delle speranze di vita futura, dell'attività svolta, delle condizioni personali e familiari della vittima.

La recente giurisprudenza di merito nel tentativo di elaborare criteri risarcitori idonei a garantire equità nella quantificazione del danno è pervenuta a risultati non omogenei :

-Tribunale di Brindisi nella sentenza n²039 del 1/12/2014 ha riconosciuto“in capo al de cuius un danno non patrimoniale, suscettibile di trasmissione agli eredi, da liquidarsi nella misura massima accordabile a fronte della compromissione dell'integrità psico - fisica, ovvero quella del 100 per cento. Questo Giudice è a conoscenza della pluralità di metodi liquidatori affermatisi nella prassi, ma ritiene che solo tal ultimo consenta di riconoscere rilievo giuridico alla perdita del bene non solo della salute, ma della vita stessa. A tale titolo, dunque, agli eredi devono riconoscersi euro 1.018.126,00, pari ad un danno biologico terminale del 100 per cento, subito da un soggetto di anni 32, nonché euro 1204.882 pari ad un danno biologico terminale del 100 per cento, subito da un soggetto di mesi 9";

-il Tribunale di Piacenza nella sentenza $n^{\circ} 97$ del 5/2/2015 ha stabilito un importo risarcitorio di $€$ 1.200 .000 a titolo di danno da perdita della vita trasmissibile agli eredi;

-la Corte di Appello di Cagliari nella citata sentenza nº 438 del 1/7/2014 aderendo alle argomentazioni espresse nella sentenza 1361/2014 ha liquidato, in ragione della giovane età del ragazzo deceduto (18 anni), € 600.000 a titolo di danno tanatologico;

-con la sentenza n⿳158 del 30/4/2014 il Tribunale del Vallo della Lucania - premettendo che è estremamente difficile dare un valore alla vita umana e che ogni forma di risarcimento pecuniario appare inadeguato rispetto all'enormità e alla tragicità della perdita della vita di un congiunto e che l'operazione è ancor più gravosa perché rimessa a un criterio equitativo che risulta di non agevole applicazione nei casi in cui non sembra essersi ancora formata una solida base giurisprudenziale di riferimento - ha liquidato la somma di $€ 450.000$, tenuto conto del fatto che la vittima aveva solo 47 anni al momento del decesso e che le sue condizioni di salute erano sostanzialmente discrete.

\section{La sentenza delle S.U. $n^{\circ} 15350$ del 22.07.15: il danno tanatologico non è risarcibile.}

Le Sezioni Unite, a seguito dell'ordinanza di rimessione $n^{\circ} 5056 / 2014$, chiamate ad affrontare il quesito circa la risarcibilità o meno del danno da perdita della vita immediatamente conseguente alle lesioni derivanti da fatto illecito, con la sentenza $n^{\circ}$ 15350/2015 hanno optato in favore della tesi maggioritaria e prevalente di segno negativo spegnendo le speranze sorte dalla coraggiosa sentenza "Scarano" $\mathrm{n}^{\circ} 1361 / 2014$.

Le ragioni su cui le S.U. hanno basato la loro statuizione - che nulla aggiungono alle argomentazioni già espresse in passato dalla prevalente giurisprudenza - sono sinteticamente le seguenti. 
Un primo aspetto riguarda la titolarità del diritto: il danno da morte non lede il bene giuridico "salute" ma il diverso bene "vita" "...fruibile solo in natura da parte del titolare e insuscettibile di essere reintegrato per equivalente"; ne consegue che il diritto al risarcimento del danno c.d. tanatologico è adespota, privo cioè di legittimo titolare, perché, nel momento in cui si concretizza il pregiudizio, l'unico legittimo titolare viene a mancare. Un altro argomento affronta il tema del richiamo operato sorprendentemente dalla sentenza "Scarano" alla coscienza sociale che non ammetterebbe nell'attualità l'irrisarcibilità del danno tanatologico. Secondo le S.U. la coscienza sociale non può assurgere a criterio legittimo per orientare l'attività dell'interprete del diritto positivo; semmai al più la coscienza sociale potrebbe rilevare come scala di valori per auspicare o meno modifiche normative.

Sulla questione poi della incomprensibilità logica indicata dalla sentenza Scarano a proposito dell'utilità economica dell'uccisione rispetto al ferimento, perché nel caso di morte non verrebbero risarciti anche i danni iure successionis diversamente dal ferimento, le S.U. hanno ritenuto tale argomento mera suggestione, di indubbia efficacia retorica, essendo "indimostrato che la sola esclusione del credito risarcitorio trasmissibile agli eredi, comporti necessariamente una liquidazione dei danni spettanti ai congiunti di entità inferiore"; tanto più che il principio "dell'integrale risarcibilità di tutti i danni non ba copertura costiturionale ed è quindi compatibile con l'esclusione del credito risarcitorio conseguente alla stessa struttura della responsabilità civile".

Un ultimo importante argomento è fondato sul rifiuto delle S.U. di accettare l'assunto come fatto dalla sentenza nomofilattica n. 1361/2014, secondo cui il danno da morte eccezionalmente sarebbe risarcibile ex se come danno evento in quanto ciò introdurrebbe un'eccezione di portata così ampia da scardinare l'intero sistema della responsabilità civile basato sul danno conseguenza ex art. 2043 c.c.

Alla luce di tali considerazioni, le Sezioni Unite con la sentenza n¹5350/2015 hanno stabilito che, nel caso di morte immediata o che segua entro brevissimo lasso di tempo alle lesioni, non possa essere invocato un diritto al risarcimento del danno iure hereditatis da parte degli eredi della vittima deceduta ad opera del fatto illecito altrui.

La dottrina più favorevole alla tesi della risarcibilità del danno tanatologico ha subito posto in evidenza i punti di debolezza presenti nella sentenza delle S.U. nº15350 che si vanno qui di seguito ad esplicitare.

\section{Osservazioni critiche sulla motivazione delle S.U. $\mathrm{n}^{\circ} 15350$.}

\subsection{Sulla carenza della titolarità.}

Non vi è dubbio che il danno da morte rientri nel novero dei danni alla persona, differenziandosene da tutti gli altri tuttavia perché colpisce uno degli aspetti della persona, il fondamentale, quello che costituisce il presupposto per l'estrinsecarsi degli altri.

Tale diversità non impedisce di ritenere che la morte si risolva in una ingiusta deminutio della persona.

La morte immediata deve essere proprio vista come lesione del diritto di vita, al di fuori della visione biologica fatta propria dalle Sezioni Unite.

Se si considera la morte immediata come lesione del bene vita in sé, allora si giunge al risultato di considerare che il danno mortale è sicuramente un danno ingiusto, poiché colpisce un diritto e cioè il diritto alla vita, riconosciuto all'art. 2 della nostra Costituzione - e presupposto delle norme penali art. 575, 
589 c.p. che incriminano l'omicidio doloso e colposo - e ribadito dall'art. 2 della Convenzione Europea dei Diritti dell'Uomo.

La giurisprudenza di merito, con particolare attenzione al tema della titolarità della pretesa al risarcimento del danno tanatologico e prendendo in esame gli artt. 2043 c.c. e 2059 c.c., sottolinea la centralità del danno rispetto ai criteri d'imputazione soggettivi, atteso che dalla produzione di un danno ingiusto deriva in primo luogo l'obbligo, per il soggetto cui quel danno sia imputabile (a titolo di colpa, dolo, responsabilità oggettiva), di risarcirlo, di pagare una somma di denaro diretta a risarcire per equivalente il danno cagionato.

La preminenza del danno rispetto al soggetto cui imputare detto danno fa sì che il destinatario del risarcimento, che pure deve necessariamente esistere, è posto in secondo piano rispetto all'obbligato e al suo obbligo di risarcimento.

Primum risarcire, deinde stabilire in favore di chi il risarcimento debba essere accordato.

Stabilire chi sia il titolare del credito risarcitorio è questione non già naturalistica, che si possa risolvere negativamente sulla base del fatto che il danneggiato ha perduto la vita, ma giuridica, da risolversi, dunque, secondo criteri analoghi a quelli che, sul versante opposto dell'obbligazione risarcitoria, conducono ad imputare la responsabilità risarcitoria di determinati danni a soggetti diversi rispetto a chi li ha materialmente prodotti.

Si aggiunga inoltre che nel campo della responsabilità civile e del risarcimento del danno da circolazione stradale si possono verificare casi in cui, alla morte di una persona, si unisca contemporaneamente e istantaneamente il danneggiamento o addirittura la distruzione, anche di cose materiali appartenenti alla vittima. In tali casi appare chiaro che, per effetto della lesione della proprietà, sorge un obbligo di risarcimento a carico del danneggiante e che il relativo credito sia trasmissibile agli eredi secondo le regole successorie ordinarie.

Nessuno può dubitare che in tali casi si assista alla contestuale lesione della situazione proprietaria e della lesione del bene-vita e al successivo sorgere di una pretesa risarcitoria che appare sin dall'origine "orfana" di un titolare, così come avviene per il credito risarcitorio commisurato alla perdita della vita.

Ne discende che la tesi della non risarcibilità del danno da morte immediata, se portata alle sue estreme conseguenze, conduce a risultati illogici se non aberranti, in quanto si fonda su una ricostruzione delle vicende giuridiche delle situazioni soggettive che non viene mai utilizzata in altri casi e che, ove lo fosse, sarebbe immediatamente rifiutata.

A questo proposito va osservato che nell'ordinamento esiste un'altra ipotesi in cui la morte di una persona fa acquistare agli eredi diritti di credito che trovano la propria fonte nel decesso del de cuius: ci si riferisce alla fattispecie dell'art. 2122, 3 comma, c.c., in cui le indennità, dovute dal datore di lavoro al lavoratore, ex artt. 2118 e 2120 c.c., per la cessazione del rapporto lavorativo senza preavviso spettano, in mancanza dei prossimi congiunti che acquistano il diritto iure proprio, agli eredi secondo le regole sulla successione legittima.

In questa ipotesi, i diritti alle indennità vengono trasmessi iure ereditario agli eredi, sebbene non vi sia un momento, neppure un brevissimo lasso temporale, in cui di tali diritti sia titolare il lavoratore. 
A livello normativo dunque esiste un meccanismo che faccia acquistare agli eredi un credito di cui il de cuius non abbia mai potuto godere e che sia corrispondente a una perdita che egli non potrà mai apprezzare.

Non si capisce quindi in tale contesto perché mai nel sistema della responsabilità civile e più in particolare della materia del risarcimento del danno alla persona non possa essere applicato il medesimo meccanismo riconoscendo la titolarità del diritto di credito risarcitorio agli eredi della vittima di un illecito ad opera di terzi, essendo stato il de cuis privato del diritto di godere della propria esistenza.

\subsection{Sul richiamo alla coscienza sociale.}

Va osservato che le Sezioni Unite affermano inoltre che non è possibile richiamare la coscienza sociale (indicata dalla sentenza "Scarano") che ha difficoltà ad accettare l'irrisarcibilità del citato danno, quale criterio legittimo per orientare l'attività dell'interprete del diritto positivo.

Per quanto sia vero che la coscienza sociale non sia un criterio interpretativo del diritto, tuttavia la coscienza sociale funge da campanello di allarme nel caso venga rilevata l'illogicità del sistema.

In altri termini non rileva certamente la coscienza sociale ex se, ma rilevano le ragioni che vengono addotte e la giurisprudenza, soggetta al vincolo motivazionale delle proprie decisioni soprattutto per merito dell'art. 111 Cost., deve tenerne conto.

Dovendo il giudice motivare i provvedimenti, allora è tenuto alla logicità, ma la logicità viene compromessa quando si nega un diritto al risarcimento di un danno tutelato dall'ordinamento.

La coscienza sociale è in questo contesto che viene correttamente richiamata.

Va infine osservato che a proposito di criteri interpretativi, l'art. 12 delle c.d. Preleggi afferma che bisogna tener conto anche dell'intenzione del legislatore.

Sostenere che il legislatore abbia voluto un sistema risarcitorio in cui è disciplinata la lesione, ma non l'uccisione è di certo una illogicità che la coscienza sociale non può che respingere.

\subsection{Sulla maggiore convenienza economica dell'uccisione rispetto al ferimento.}

Le Sezioni Unite ritengono non dimostrato il fatto che sia economicamente più conveniente uccidere che ferire, rispetto all'attuale assetto risarcitorio.

Per gli ermellini, contrariamente alle argomentazioni della sentenza "Scarano", è logicamente dimostrato che se si sottrae una voce risarcitoria, soprattutto quella qualificata come danno morale terminale o psichico terminale, allora il quantum debeatur complessivo si abbassa e, se si sottrae, il risultato è di certo inferiore rispetto ai casi di sommatoria.

A tale affermazione è ragionevole far osservare che non bisogna guardare il singolo pregiudizio perché alcuni da soli hanno un quantum più alto rispetto alla sommatoria di "piccoli" pregiudizi. Nell'ipotesi di danno tanatologico si parla della lesione del bene vita, ovvero del bene giuridico più prezioso in assoluto che viene sottratto: non vengono, cioè, sottratti "piccoli" pregiudizi, ma probabilmente il bene più importante in assoluto.

\subsection{Sulla risarcibilità del danno tanatologico come danno evento.}


Le Sezioni Unite sostengono che non sia possibile risarcire il danno evento, neanche in via eccezionale, perché in tal caso si tratterebbe di un'eccezione capace di vulnerare l'intero sistema.

Parte della dottrina dissente sul punto osservando che la risarcibilità del solo danno conseguenza discende innanzitutto dall'art. 1223 c.c. laddove si parla di "conseguenza immediata e diretta".

Nel caso di decesso della vittima per fatto illecito altrui l'evento mortale coincide con la conseguenza della perdita della vita e pertanto il c.d. danno evento ed il c.d. danno conseguenza si fondono, così rendendo applicabile l'art. 1223 c.c. senza compromettere la stabilità del sistema risarcitorio elaborato dalla giurisprudenza.

Infatti se si giunge a concludere che nel caso di perdita della vita danno evento e danno conseguenza coincidono, allora non siamo di fronte ad un'eccezione al principio dell'irrisarcibilità del danno evento perché, si sta risarcendo un danno conseguenza; tenendo conto delle peculiarità del danno tanatologico rispetto ad altri pregiudizi. E facendo salva la previsione di cui all'art. 1223 c.c. senza operare alcuna deroga.

\subsection{In conclusione.}

Alla luce di tutto quanto sopra considerato pare doveroso segnalare che il dictum delle S.U. non è idoneo a chiudere definitivamente la questione e ad arrestare il cammino dei fautori della tesi favorevole al risarcimento del danno da morte immediata, non essendo la stringata motivazione delle S.U. convincente e risolutiva rispetto alle approfondite e coraggiose argomentazioni della sentenza Scarano n. 1361/2014, che delineava la risarcibilità ex se del bene vita, oggetto di un diritto assoluto ed inviolabile.

\section{Riferimenti bibliografici.}

- Buffone G., "Il risarcimento del danno non patrimoniale ossia ildecimo problema di HIlbert", in Responsabilità Civile e Previdenza, fasc. 3, 2015, pp. 1007B e ss.

- Giusti M., "Giurisprudenza sul danno biologico e morale terminale: natura e liquidazione", in Responsabilità Civile e Previdenza, fasc. 3, 2015, pp. 892 e ss.

- Navarretta E., "La 'vera' giustizia e il 'giusto' responso delle S.U. sul danno tanatologico iure ereditario", in Responsabilità Civile e Previdenza, fasc. 5, 2015, pp. 1416B e ss.

- Savoia R., "Le Sezioni Unite confermano l'irrisarcibilità del danno tanatologico", in Diritto \& Giustizia, fasc. 29, 2015, pp. 59 e ss.

- Ziviz P., "Illusioni perdute", in Responsabilità Civile e Previdenza, fasc. 5, 2015, pp. 1443 B e ss. 